\title{
Proceedings of the meeting of the Association of British Neurologists, University Hospital, Nottingham, 7-9 April 1988
}

INTRAVENOUS METHYLPREDNISOLONE VERSUS INTRAMUSCULAR ACTH IN THE TREATMENT OF ACUTE RELAPSES IN MULTIPLE SCLEROSIS MS Methylprednisolone Study Group, The London, Charing Cross and Guy's Hospitals, London; North Manchester General Hospital, Manchester; Pinderfields Hospital, Wakefield

It has been suggested that high dose intravenous (iv) methylprednisolone is useful in the treatment of relapse in multiple sclerosis (MS). Since it has been demonstrated that intramuscular (im) ACTH is effective in the management of relapse a randomised double-blind controlled trial was undertaken to compare iv methylprednisolone with im ACTH in 61 patients with clinically definite MS. All patients were in acute relapse (seen within 4 weeks of the onset of symptoms) and were randomised to one or other treatment regimen: (1) $1 \mathrm{~g}$ methylprednisolone daily for 3 days, (2) ACTH, initially at 80 iu daily for 5 days, decreasing gradually over the subsequent 9 days.

Patients were assessed clinically according to Kurtzke's disability status and functional system scales immediately prior to treatment and on days, 3, 7, 14, 28 and 3 months after treatment.

There was no difference in age, sex, age of onset, duration and severity of disease or severity of relapse between the two groups. The results of the study were assessed using linear trend analysis, Student's $t$ test and the Mann-Whitney rank sum test. Both groups showed significant improvement during the course of the study. There was no significant difference between the two groups in either the rate of recovery or the level of improvement at 3 months. Thus iv methylprednisolone is a useful alternative in the management of acute relapse in MS.

CHILDHOOD ONSET MULTIPLE SCLEROSIS

DH Mellor. University Hospital, Nottingham

Four children have been diagnosed as having multiple sclerosis (MS) in South Trent over the last 3 years. The age at first symptoms varied from 7-12 years. One child presented with a subacute encephalopathy but later had recurrent episodes of ataxia. All the other three children had a gait disturbance at presentation with both spasticity and ataxia. Episodes of optic or retrobulbar neuritis occurred in two children. Two children had transient facial palsies, one at presentation and the other during a relapse. All four children have had a relapsingremitting course.

Abnormalities were seen on CT in only two children at presentation, and the appearances were misleading in one. In one child CT repeated after several relapses showed typical changes of MS. One child had a normal initial CT scan but MRI showed patchy demyelination throughout both hemispheres.

VERs were abnormal in three children at presentation and in all children at some time during the course of the illness. CSF at presentation showed oligoclonal bands in two children and a third became positive after several relapses. All the children were treated with ACTH following diagnosis and all had a good response.

MS commencing before 13 years accounts for only $0.5 \%$ of all cases of MS but the diagnosis may have to be considered in a wide variety of childhood neurological illnesses. MRI scanning is of particular diagnostic value but monophasic "postinfectious" demyelination may be difficult to exclude at presentation.

\section{CHILDHOOD OPTIC NEURITIS}

DA Francis, A Kriss, F Cuendet, DSI Taylor, JR Batchelor, WI McDonald. The National Hospitals for Nervous Diseases and Hospital for Sick Children, London

The visual prognosis, recurrence rate and risk of multiple sclerosis (MS) after childhood optic neuritis (ON) remain uncertain. We have investigated these questions by reexamining 39 children, who presented with $O N$, on average 9 years later.

Girls predominated ( $75 \%)$; average age at presentation 8.6 years; 25 children had both eyes affected simultaneously (symptoms in second eye within 2 weeks); 14 children had unilateral or bilateral sequential episodes $\mathscr{\varphi}$ with involvement of the second eye in $2-4$ 을 weeks. The overall prognosis for visual func- 흘 tion was excellent $\left(6 / 9\right.$ or better in $80 \%$ of $\frac{\bar{\sigma}}{\partial}$ children with VEPs returning to normal in $\mathbb{D}$ $55 \%)$. Only four patients $(10 \%)$ had recurrent episodes and six children (15\%) sub- $\oplus$ sequently developed MS ( 2 patients CDMS; $\overrightarrow{0}$ 4 patients CPMS); one followed recurrent ON. A peak incidence in Spring (36\%) and $\vec{\omega}$ high association with febrile onset suggested $\sigma$ an infectious basis in most patients.

Optic neuritis in children differs from the adult form in the lower recurrence rates, of reduced risk of MS and higher frequency of VEP recovery despite the presence of optie ? atrophy. This suggests a greater potential for $\vec{\omega}$ remyelination in childhood. No factors (age, $\mathrm{G}$ sex, etc) for the later development of $\mathrm{MS}$ 응 were identified, although unilateral case $\supset$ carrying MS-associated HLA antigen seemed at risk.

HYSTERIA PRESENTING TO A PAEDIATRIC NEUROLOGY DEPARTMENT MA Clarke, MJ Noronha. Booth Hall Chitdren's Hospital, Manchester

The fear of missed organic disease and difficulties in definition led Eliot Slater to describe a diagnosis of hysteria as a disguise for ignorance and a fertile source of clinical $\frac{\circ}{\not}$ error.

Recent ideas of sick-role and illness $\overrightarrow{\bar{O}}$ behaviour have emphasised individual vari- $\frac{3}{3}$ ation in the perception of symptoms and the $\bar{Z}$ ways in which abnormal illness behaviour may be learned in a medical setting.

Twenty-three children (median age $13 \overline{\bar{\partial}}$ years) were referred by other consultants to $\frac{}{0}$ this paediatric neurology department with apparently serious neurological disorder. Sixteen had an abnormal gait or paraplegia, $ᄋ$ four visual deficit, and torticollis, ptosis and 3 tremor in one child each. Eighty per cent had 0 a preceding flu-like illness or minor injury. None had neurological disease and only one $\frac{D}{O}$ a previous psychiatric history. Nineteen were "high achievers", at the top of their N class, aspiring to professional careers and 
often receiving extra home tuition. Two other children had been sexually abused and one had unresolved grief. In one child no cause was found.

Twenty recovered with physiotherapy and psychiatric intervention which emphasised the ways in which learned responses to symptoms could result in abnormal illness behaviour when a child was under more continuous stress, usually at school.

\section{FAMILIAL NON-HUNTINGTON'S CHOREA}

J Meara, W Schady. Manchester Royal Infirmary, Manchester

Hereditary chorea without dementia is a rare disorder characterised by the onset of choreiform movements in early childhood. Autosomal dominant inheritance is usual, but normal intellectual development and the absence of rigidity, seizures or ataxia distinguish it from juvenile Huntington's disease. The involuntary movements are said not to progress beyond the third decade and may even improve thereafter.

We describe a family in whom there is clear evidence of progression in severity of the disorder with time, resulting in considerable disability, though longevity and intellect have not been affected. Inheritance was autosomal dominant. Chorea began in the first decade of life, with subsequent development of facial grimacing, dysarthria and pyramidal signs. In addition, two family members exhibited dystonic posturing, a feature not previously described. The oldest affected member died at the age of 100 , while two others are alive aged 67 and 75 years. However, their gait is markedly disturbed and dyskinesia interferes severely with speech and limb function.

Previous reports have dealt with young individuals early on in the disease. Our family shows that hereditary nonHuntington's chorea may progress throughout life, possibily accelerated by agerelated loss of striatal neurons. The term "benign" should therefore be avoided.

CORTICAL NEUROTRANSMITTERS IN ALZHEIMER'S AND PICK'S DISEASES GP Reynolds, K Mercer, J Lowe. University of Nottingham Medical School, Nottingham

As part of a major study of neurotransmitter function in dementing disorders, we have measured concentrations of monoamine and amino acid transmitters in frontal and temporal cortex taken post mortem from three cases of Pick's disease, six of Alzheimer's disease and six control subjects. The controls had no history of neurological or psychiatric disorder, and the two dementia groups were defined by one of us (JL) using established neuropathological criteria. The groups were approximately matched for age and sex.

Both frontal and temporal regions showed deficits for $\gamma$-aminobutyric acid (GABA) in Alzheimer's disease $(75 \%$ and $64 \%$ of control values respectively) and in Pick's disease $(69 \%$ and $60 \%)$. Losses of noradrenaline and serotonin, but not dopamine, were apparent in the temporal cortex in Alzheimer's disease, confirming several previous reports. However, no indication of any loss in these neurotransmitters was apparent in either region of the Pick's disease patients.

We conclude that while Pick's and Alzheimer's diseases share a loss of intrinsic cortical neurons (such as those containing GABA), Pick's disease does not exhibit the loss of cortical projections from brain stem nuclei that occurs in Alzheimer's disease.

CORTICAL LEWY BODIES AND THE SPECTRUM OF LEWY BODY DISEASE

G Lennox, J Lowe, J Byrne, G Reynolds, RB Godwin-Austen. University Hospital, Nottingham

The nosological status of cortical Lewy bodies (CLB) in Parkinson's disease and dementia remains controversial. We describe the clinical and pathological features of the 15 cases in which CLB were identifiediat postmortem during one year.

The mean age of onset was 72 years (range 62-84) and the mean duration of illness was 5 years (range 1-19). In contrast to previous reports, $6(40 \%)$ presented as typical Parkinson's disease without cognitive impairment, $3(20 \%)$ with both Parkinsonian features and cognitive impairment and only $6(40 \%)$ with cognitive impairment alone. In time, all $15(100 \%)$ came to show both Parkinsonian features (generally dominated by rigidity and changes in posture) and progressive global dementia (generally with cortical features such as dysphasia).

All cases had Lewy bodies in brainstem nuclei and cerebral cortex. CLB were most frequent in the anterior cingulate, insular, temporal and frontal cortices, affecting up to $4 \%$ of neurons in these areas. Numbers of CLB correlated with degree of dementia. In 9 cases there were no other pathological changes; in 6 cases there were also small numbers of neurofibrillary tangles.

Preliminary neurochemical studies reveal substantial $(>90 \%)$ reductions of dopamine and HVA levels in basal ganglia regardless of mode of presentation.

We suggest that Lewy Body Disease
(LBD) may be the pathological substrate of a spectrum of neurological disorders, $\bar{Z}$ including the dementia of Parkinson's disease.

SKELETAL MUSCLE CHANGES IN ACROMEGALY RE Jones, SM Bruce, JAG Punt, J Lowe University Hospital, Nottingham

Endocrine myopathies associated with disorders of thyroid hormone or cortisol secretion are well recognised and described. Less frequently studied, however, is the myop? athy associated with acromegaly, even though muscular complaints are common in this condition.

We have studied muscle biopsies in $10 \mathbb{8}$ patients with acromegaly at the time of transphenoidal hypophysectomy. Three types of histological abnormality werẹ found:

(1) Type 1 hypertrophy with atrophy of Type 2 fibres. Of the six specimens in this group, three also showed hyper trophy of some Type 2 fibres. The atro? phic fibres were typically of subgroup 2B. The five most atrophic samples con $\vec{\circ}$ tained evidence of fibres splitting. (2) Three biopsies had atrophy of the T Tw 2 fibres without concomitant Type hypertrophy.

(3) In one sample, there was generalised? fibre hypertrophy involving both fireo types without any other abnormalits

Quantitative (morphometric) analysisgo fibre cross sectional area was also performired on all the samples. Seven of the 10 biopsy specimens showed fibres of abnormal crosso sectional area when analysed according to strict morphometric criteria.

\section{ACETYLCHOLINE RECEPTOR-SPECIFIC}

T LYMPHOCYTE RESPONSES IN THYMUS, THYMOMA AND LYMPH NODES FROM PATIENTS WITH MYASTHENIA GRAVIS N Sommer, G Harcourt, $N$ Willcox, $\frac{F}{5}$ Newsom-Davis. Radcliffe Infirmary, Oxford

Thymic abnormalities are among the most consistent features in myasthenia gravis. Cel토 preparations from 32 hyperplastic thymuses 4 thymomas and 10 lymph nodes from MGo patients undergoing thymectomy have been? tested for their $\mathrm{T}$ cell reactivity $(3 \mathrm{H}$ ? thymidine uptake) to acetylcholine receptor (AChR) from electric organ of Torpedo mar- $^{-}$ morata.

Cell suspensions from hyperplastic thy muses responded in $55 \%$ of cases. Stimuㅡㅡㄹ lation indices (SI) were between $2 \cdot 1$ and $6 \cdot 5 \mathrm{~N}$ and did not correlate with the anti-AChR 


\section{Proceedings of the meeting of the Association of British Neurologists}

antibody titre in serum. Seven out of 10 mediastinal lymph nodes from these patients showed responses between 2.4 and 27.2 . Also, in all 4 thymoma cases, both thymoma and uninvolved thymus were positive (SI 2.1-20.8).

By contrast, only $32 \%$ of these patients showed positive responses in peripheral blood; SI were lower than in thymus in $83 \%$ of cases, whereas for a typical "recall" antigen (tuberculin ppd) they were higher in blood in $94 \%$.

We conclude that AChR-specific T cells can be detected more consistently in thymus and lymph node than in blood, according with the hypothesis that the autoimmune process in MG is sustained by $\mathrm{T}$ cells chronically generated in the thymus.

THE AETIOLOGY OF TRANSIENT GLOBAL AMNESIA (TGA): A CASE-CONTROL STUDY OF 114 CASES

JR Hodges, CP Warlow. Radcliffe Infirmary, Oxford

The aetiology of TGA is unsettled. Most authorities consider it to be a form of transient ischaemic event. Our provisional study of 50 retrospectively ascertained cases found no evidence to support a cerebrovascular aetiology.

We have now completed a case-control study, with prospective follow-up, of 114 TGA cases, 109 age/sex matched normal (community) controls and 212 matched TIA controls.

The prevalence of vascular risk factors (hypertension, ischaemic heart disease, carotid bruits, smoking, polycythaemia) was not significantly different in TGAs and normal controls, whereas their prevalence was significantly greater in TIA controls than TGAs. The only factor associated with an increased risk of TGA was migraine.

Follow-up of TGAs and TIA controls revealed a very different outcome. Of the 114 TGAs, two have had major vascular events (stroke, myocardial infarct or vascular death); adjusted event rate: $613 / 100,000$ patient years. By contrast, of the 212 TIAs, 49 have had such events $(6698 / 100,000$ patient years). Eight TGA patients (7\%) have manifest epilepsy.

In conclusion, thrombo-embolic cerebrovascular disease is not the cause of TGA. Migraine may play a part. An important minority of cases eventually manifest epilepsy.
THE EFFECT OF EPHEDRINE ON CEREBRAL BLOOD FLOW AND MIDDLE CEREBRAL ARTERY VELOCITY IN PATIENTS WITH ORTHOSTATIC HYPOTENSION

DJ Brooks, S Redmond, R Bannister, C Matthias, L Symon. Hammersmith and National Hospitals for Nervous Diseases, London

Cerebral blood flow (CBF) and middle cerebral artery velocity (MCA vel) have been measured in four patients with pure autonomic failure and four patients with multisystem atrophy using ${ }^{133} \mathrm{Xe}$ washout and transcranial doppler. CBF and MCA velocity measurements were performed both with the patients lying flat and with the patients tilted to a degree that resulted in a significant fall in their blood pressure (BP) without inducing syncope. These measurements were then repeated one hour after the patients had taken a $30 \mathrm{mg}$ oral dose of ephedrine.

The eight patients showed normal autoregulation, no significant change in their CBF occurring when they were tilted. Hypotension however was accompanied by a fall in MCA velocity suggesting that dilatation of the middle cerebral artery was occurring in order to maintain CBF by reducing arterial resistance. After taking ephedrine there was a rise in lying and tilted BP and MCA velocity, but no significant change in CBF. This finding suggests that ephedrine increases BP via inducing vasoconstriction, but has no direct effect on cerebral blood flow. As such, its beneficial action in patients with orthostatic hypotension is probably related to its ability to maintain tilted BP within the autoregulatory range, rather than by any direct action on CBF.

Two patients with orthostatic hypotension secondary to dopamine- $\beta$ hydroxylase deficiency were also studied. These patients had negligible plasma levels of noradrenaline and adrenaline. Ephedrine led to a paradoxical fall in BP and CBF in these patients suggesting that $\beta$ rather than the usual $\alpha$ sympathomimetic effects were predominating.

\section{CT, MRI AND SPECT IMAGING IN CORTICAL} BLINDNESS DUE TO ECLAMPSIA

R Duncan, J Patterson, D Hadley, I Bone, EM Symonds, BS Worthington, PC Rubin. Southern General Hospital, Glasgow

Blindness is a rare complication of eclampsia. It is usually due to lesions in the occipital cortex which show as areas of low attenuation on computed tomography (CT). We have studied three patients with blind- ness due to eclampsia using CT, magnetic 으 resonance imaging (MRI), and single $Z$ photon emission computed tomography $\stackrel{\mathbb{C}}{=}$ (SPECT). We present clinical case reports and a review of previous cases.

In one case unenhanced CT showed low $\overline{\widehat{\theta}}$ density occipital lesions, and in two cases $T$ was normal. MRI showed long T1 and T2 occipital lesions in all three cases. In one case MRI also showed a clinically silent long T1 률 and T2 lesion in the white matter of the left temporal lobe. SPECT carried out on this patient showed hypoperfusion in both occip- $\stackrel{\vec{P}}{+}$ ital cortices and also showed the temporal $\bar{C}$ lesion to be hypoperfused with surrounding $\frac{\bar{\sigma}}{\bar{D}}$ hyperperfusion. Follow-up MRI and $\overline{\bar{C}}$ SPECT at 6 weeks showed near complete $\frac{\nabla}{\Phi}$ resolution of all lesions.

Pathological studies have suggested hae- ळ) morrhage rather than ischaemia as a cause $\overrightarrow{0}$ for the lesions in eclampsia; however, the coincidence of long $T 1$ and T2 lesions on $\vec{\omega}$ MRI and hypoperfused lesions on SPECT with surrounding hyperaemia indicate that the lesions seen were ischaemia.

\section{PROGNOSTIC FACTORS IN ACUTE STROKE; THF OXFORDSHIRE COMMUNITY STROKE PROJECT PAG Sandercock, JM Bamford, M Dennis J Burn, L Jones. Western General Hospita Edinburgh}

Six hundred and seventy-five patients with first-ever stroke have been registered by the Oxfordshire Community Stroke Project. Follow-up data to $\mathbf{3 0}$ days after stroke onset are now complete. Univariate analysis showed that the presence of any one of the following factors was associated with a statistically significant $(p<0.05)$ increase in $\frac{2}{D}$ 30 day case-fatality rate: subarachnoid $\varrho$ haemorrhage, primary intracerebral hae- $\overrightarrow{\overrightarrow{0}}$ morrhage, total anterior circulation 3 infarction, impaired consciousness at $24 \mathrm{hrs} \rightleftharpoons$ after stroke onset, atrial fibrillation, a cardiac lesion considered a "major" potential source of embolism to the brain and increasing age. Factors associated with a significantly reduced 30 day case fatality rate were: partial anterior circulation infarction, posterior circulation infarction, lacunar $ᄋ$ infarction, and symptoms or signs of atheromatous vascular disease. These findings are 0 likely to be important for clinical research when attempting to identify homogenous $\frac{7}{0}$ groups of patients (with similar causation and natural history) and when designing $N$ treatment trials in acute stroke. 
CHRONIC PROGRESSIVE PERIPHERAL NEUROPATHY RESTRICTED TO THE ARMS M Donaghy, J Gawler, J Winer, S Liebowitz, RHM King, PK Thomas. The Royal Free, St Bartholomew's, Guy's, and The National Hospitals for Nervous Diseases, London

Seven patients had chronic progressive peripheral neuropathy restricted to the arms. Radial nerve biopsies from four patients showed nonspecific axonal loss. Sensory action potentials were reduced and muscles denervated in the arms; nerve conduction was normal in the legs. Neither the clinical picture nor the investigations implicated vasculitis, tumour infiltration of the brachial plexus or acute idiopathic brachial plexus neuropathy as causes of the neuropathy. Only one arm was affected in the three younger patients aged $32-44$ years in whom there was no underlying neoplasm. Both arms were affected in the four older patients aged 50-79 who all had underlying neoplasms which were anatomically unrelated to the brachial plexus. Two of these patients had an IgMk paraproteinaemia without anti-myelin associated glycoprotein activity or evidence of demyelinating neuropathy. We conclude that there is a clinical syndrome of chronic progressive idiopathic upper limb neuropathy which, if bilateral, may be a paraneoplastic phenomenon.

\section{UBIQUITIN IMMUNOREACTIVITY IN}

NEURODEGENERATIVE DISEASE

J Lowe, G Lennox, RJ Mayer, K Morrell, M Landon, RB Godwin-Austen. University Hospital, Nottingham

Ubiquitin is a 76 residue protein found in abundance throughout the plant and animal kingdoms and is the most highly conserved protein through evolution. It is produced by cells in response to a wide variety of cellular stresses (including heat shock, toxins and viral infection), and has a role in mediating intracellular proteolysis. Antibodies to ubiquitin have been used to localise ubiquitin in histological sections by immunocyto-chemistry from cases of Alzheimer's disease, Parkinson's disease, Pick's disease, Dementia pugilistica and motor neuron disease. Ubiquitin immunoreactivity is localised to the fibrillar inclusions which are the histological hallmarks of these disease processes (tangles, Lewy bodies, Pick bodies).

Immunocytochemical staining with ubiquitin shows intraneuronal inclusion bodies, such as tangles and Lewy bodies, with such clarity that it can be regarded as a new stan- dard for their detection. This is particularly evident for cortical Lewy bodies.

These observations show that abnormal ubiquitin activation is a common factor in several neurodegenerative diseases. Ubiquitin may be acting in a cyto-protective manner by targeting either abnormal or damaged proteins for proteolysis. Overload of this system would explain accumulation of ubiquitinated protein inclusion bodies in these disease states. Further understanding of the role of ubiquitin in the formation of inclusion bodies will throw light on the pathogenesis of the disease processes.

\section{UBIQUITIN-IMMUNOREACTIVE DEPOSITS IN} MOTOR NEURON DISEASE ANTERIOR HORN CELLS

PN Leigh, BH Anderton, A Dodson, M Swash, D Power. St George's and The London Hospitals, London

Ubiquitin is implicated in the non-lysosomal degradation of short-lived and abnormal proteins and has been identified as a component of Paired Helical Filaments (PHF) in Alzheimer's disease tangles, suggesting that ubiquitinated proteins in PHF are resistant to removal via the ubiquitin pathway. Since cytoskeletal abnormalities also occur in MND, we used immunocytochemistry to examine spinal cord tissue from 10 MND cases, and 12 controls with a polyclonal antibody against ubiquitin. In all MND cases, but in none of the controls, we observed previously unrecognised anterior horn cell cytoplasmic inclusions. These took the form either of densely staining masses $(5-10 \mu \mathrm{m}$ diameter), or aggregations of filamentous "skeins".

These inclusions did not correspond to Bunina bodies, or to other inclusions seen in MND anterior horn cells. In addition, $30 \%$ of axonal spheroids in normal and in MND tissue showed punctate ubiquitin positive staining. We interpret these findings to suggest that conjugation of abnormal proteins to ubiquitin occurs in anterior horn cells in MND, and spheroids in normal and MND tissue. Identification of the protein(s) to which ubiquitin is conjugated in anterior horn cells will provide new insights into pathogenic mechanisms in MND.

\section{RESPIRATORY COMPLICATIONS AND THEIR}

MANAGEMENT IN MOTOR NEURON DISEASE RS Howard, L Loh, CM Wiles. The National Hospitals for Nervous Diseases, London

Respiratory insufficiency usually develops in the advanced stages of motor neuron disease and is the commonest cause of death. How $Z$ ever, some patients may present with acute respiratory insufficiency or develop distressing respiratory symptoms early in the course of the disease.

We present 14 patients with motor neurongs disease who were considered for respiratory support. Eleven of these patients received such support and all derived significan symptomatic improvement without dis-: tressing prolongation of life.

Of the eight patients with amyotrophic ${ }^{\stackrel{5}{+}}$ lateral sclerosis seven had predominante diaphragm weakness and one generalised $\overline{\bar{O}}$ respiratory muscle weakness. Seven received $\frac{\bar{\rho}}{\widehat{D}}$ negative pressure ventilation by cuirass ${ }^{\circ}$ which improved both the quality of sleepen and exercise tolerance.

Three patients with predominantly bulbar $\overrightarrow{0}$ disease had nocturnal apnoea or hypo- ventilation. Two patients received no sup- $\vec{\omega}$ port whilst one, who also developed? diaphragm weakness, was treated by cuirass, $\bar{\partial}$ continuous positive airway pressure (CPAP) and later nocturnal intermittent positive ${ }_{-}$ pressure ventilation (IPPV).

Three patients with progressive muscuterr atrophy had predominant diaphragm wea $-\vec{\omega}$ ness or noctural apnoea. These patiefs $s$ received nocturnal CPAP, cuirass or IPBVO with symptomatic benefit.

Some patients with motor neuron diseag, mainly those with symptoms due to respigatory muscle weakness in the absence 010 severe bulbar impairment, derive beneiti from supported ventilation. However, issues involved need to be discussed, if pos- $-\infty$ sible, with the patient and relatives prior to. the development of a respiratory crisis.

PROPHYLACTIC ANTI-CONVULSANT DRUGS IN THE PREVENTION OF EPILEPSY FOLLOWING CRANIOTOMY FOR HIGH RISK BENIGN CONDITIONS

D Chadwick, N Rajagopalan, P Foy, MDM $^{3}$ Shaw. Walton Hospital, Liverpool

Craniotomy, for a variety of pathologies, carries with it a significant risk of the development of de novo epilepsy. A prospective study has been undertaken in a group of high 3 . risk patients with non-progressive pathologies who were randomised to no treatment, $\frac{\text { o }}{3}$ 6 months treatment or 2 years treatment. Therapy was commenced 24 hours prior to을 surgery. Two hundred and seventy-six $D$ patients have been followed up for a mean of을 31 months. One hundred and forty-nine had intracranial aneurysms, 50 had meningi- $\sigma$ omas, 52 other benign tumours and $25 \mathrm{~N}$ 
AVM, spontaneous haematoma or cerebral abscess.

The probability of remaining seizure free for 4 years following craniotomy was $59 \%$ for patients receiving no prophylactic treatment, $66 \%$ for those receiving 6 months treatment and $62 \%$ for those receiving 2 years treatment. These differences are not statistically significant. In addition, $11 \%$ of the drug treated group (receiving phenytoin or carbamazepine) suffered acute drug reactions including skin rash. There is no evidence from this study that prophylactic treatment influences the long term natural history of epilepsy following craniotomy.

This study may have wider implications for policies on the early treatment of epilepsy.

THE RELEVANCE OF CT HEAD SCAN IN EVALUATION OF PATIENTS WITH EPILEPSY A Esack, WJK Cumming. Withington Hospital, Manchester

District physicians frequently enquire as to whether all patients presenting with epilepsy require CT of the head.

To answer this question, 365 consecutive patients presenting to one neurological unit were investigated according to a standard protocol. All patients, in addition to the epilepsy history, had a detailed clinical examination, a standard 16 channel EEG and CT head scan. Seizure type was sub-divided into primary generalised, simple-complex partial and focal motor/sensory.

Overall, CT was abnormal in $22 \%$. CT was abnormal in $10 \%$ of primary generalised, $24 \%$ of simple-complex partial and $27 \%$ of focal motor/sensory. The frequency of abnormal examination in patients with abnormal scans were $50 \%$ for primary generakised, $40 \%$ for simple-complex partial and $93 \%$ for focal motor/sensory. In all but one patient where the CT abnormality was of significance in respect to further management, the physical examination was abnormal.

The EEG was not of predictive value in defining a clinically significant lesion on CT except in those patients with simple-complex partial and focal motor/sensory epilepsy who had gliomas when EEG was uniformly abnormal.

There was no increase in significant CT abnormalities with advancing age.

This study suggests that an abnormal physical finding is the best predictor of a clinically significant CT abnormality and that CT is not routinely required in the evaluation of all patients with epilepsy.
THE EFFECT OF REMOVAL OF ONE ANTIEPILEPTIC DRUG (AED) ON CONCOMITANT AED LEVELS JS Duncan, SD Shorvon, PN Patsalos. Chalfont Centre for Epilepsy, Buckinghamshire

Little is known about the effects of withdrawal of individual antiepileptic drugs (AEDs) on the serum concentrations of concomitant AEDs. We have determined the total and free serum AED concentrations in a prospective study of 70 patients who had phenytoin (PHT), carbamazepine (CBZ) or sodium valproate (VPA) withdrawn as part of a planned rationalisation of their drug treatment. AED concentrations were measured weekly, before and during drug reduction, and for the 4 weeks after the end of drug withdrawal. The principal findings were:

1. Phenobarbitone (PB) levels fell by a mean of $30 \%$ on removal of PHT.

2. Total CBZ levels rose by a mean of $48 \%$ on removal of PHT, with no change in CBZ-epoxide levels.

3. VPA levels rose by a mean of $19 \%$ on removal of PHT, and by a mean of $42 \%$ on removal of $\mathrm{CBZ}$.

4. PHT levels fell by a mean of $26 \%$ on removal of CBZ.

5. Total CBZ levels rose by a mean of $10 \%$ on VPA removal, with a concomitant mean $24 \%$ fall in total CBZ-epoxide levels.

The magnitude of these observed changes may be of considerable clinical importance and underlines the need for serum level monitoring when drug changes are made in clinical practice. For AEDs other than PB, serum levels one week after the completion of a drug withdrawal gave a reliable guide to the new steady state, but PB levels were not stable at this time. In general, free drug levels did not provide clinically useful information over and above that obtained from total drug levels.

\section{GABAPENTIN IN PARTIAL EPILEPSY}

J Andrews, D Bates, P Bolel, N Cartilage, D Chadwick, P Cleland, $\mathbf{P}$ Crawford, K Cumming, K Kennedy-Young, R Knight, A McKenna, P Newman, V Patterson, M Saunders, D Shephard, G Venables, G Yuill, G Boddie. Walton Hospital, Liverpool and elsewhere

Gabapentin is a gaba-analogue with anticonvulsant properties in animals. Preliminary studies in humans demonstrated anti-epileptic properties with little observed toxicity. A multi-centre parallel group placebo controlled study of Gabapentin $\frac{\text { 을 }}{Z}$ (1200 mg per day) as add on therapy was $\overparen{D}$ undertaken.

One hundred and twenty-eight patients with drug resistant partial epilepsy (min- $\frac{C}{0}$ imum one seizure per week despite therapy 0 with one or two standard anticonvulsant drugs), have now completed the double $\frac{\rho}{\partial}$ blind phase of this study. A preliminary analysis of data from the first 96 patients completing the study demonstrated that 3 months treatment with Gabapentin resulted $\overline{\vec{\sigma}}$ in $28 \%$ median reduction in partial seizures -0 compared with 3 months base line, whereas $\frac{C}{O}$ the placebo response was only $9 \%$. Reduc- $\frac{\bar{C}}{\bar{D}}$ tion of seizure frequency of greater than or $\frac{\vec{D}}{D}$

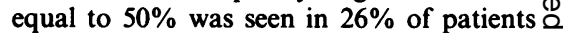
who received Gabapentin and $10 \%$ of placebo patients.

Gabapentin was well tolerated with $52 \%$. of patients reporting mostly mild to mod- $\overrightarrow{\vec{\omega}}$ erate adverse events compared to $31 \%$ on placebo. No adverse event was considered definitely related to Gabapentin. Three patients were withdrawn from each treat- ir ment group for adverse events. No inter- $\rightarrow$ actions were observed between Gabapentin $\overrightarrow{0}$ and background anti-convulsants.

Present results in this group of severes epileptic patients suggest that Gabapentim may be a useful addition to currently ava迟윽 able anti-epileptic drugs.

PROTEIN AND GLYCOPROTEIN ABNORMALITIE OF BRAIN TUMOURS FROM TUBEROUS SCLEROSIS PATIENTS

ZK Ellis, FW Hemming. University of $\infty$ Nottingham Medical School, Nottingham

Tuberous sclerosis (TS) is an autosomal dominant disease with a high rate of sporadic mutation. The characteristic feature of

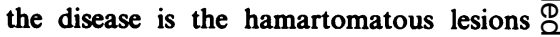
affecting almost all organs. Brain of TS patients shows striking changes. Tubers on $\frac{}{3}$ the cortex and deep lesions within the ventricular system are often seen in TS patients.

We have investigated the protein and glycoprotein pattern and content of brain tumours and brain of TS patients and com- $\frac{7}{0}$ pared them with those of normal. This has been done by polyacrylamide gel electro- 3 phoresis and Western blotting of the $\hat{\rho}$ delipidated brain tissue. Proteins were $\frac{0}{3}$ stained with Coomassie blue and glycoproteins with specific lectins. Gly- 을 copeptide composition was studied after $D$ enzymic digestion of glycoproteins followed 을 by Con A Sepharose column chro- $N$ matography. Carbohydrate content of the $\Omega$ glycopeptides was measured by gas liquid $N$ 
chromatography following acid hydrolysis.

Results indicated significant differences in the protein profile of brain tumours compared to that of normal. The overall glycoprotein profile is also different in tumours. The glycopeptide carbohydrate composition does not show significant alterations. The possible significance of these findings to the pathogenesis of TS is discussed.

REDUCED SODIUM PUMP ACTIVITY IN SCIATIC NERVES FROM DIABETIC RATS; IMPLICATIONS FOR DIABETIC NEUROPATHY

JN Hawthorne, CMF Simpson. University of Nottingham Medical School, Nottingham

Six weeks after induction of streptozotocin diabetes, ouabain-sensitive ${ }^{86} \mathbf{R b}{ }^{+}$accumulation, which reflects sodium pump activity, was significantly reduced in endoneurial preparations of sciatic nerve from diabetic rats compared with controls. The decrease was not seen when diabetic rats were maintained on sorbinil (an aldose reductase inhibitor) or myo-inositol diets. Both these diets restore the lowered inositol concentration in nerves from diabetic rats to normal. Inositol lipid metabolism provides diacylglycerol to activate protein kinase $\mathrm{C}$ and it has been suggested that this kinase controls the sodium pump. Preliminary results show that the kinase is less active in nerves from diabetic animals, but we have no evidence that the protein of the sodium pump ATPase is phosphorylated.

TRYPTOPHAN METABOLISM AND QUINOLINIC ACID IN THE BRAIN OF HUNTINGTON'S DISEASE

SJ Pearson, GP Reynolds. University of Nottingham Medical School, Nottingham

While there have been substantial advances in our understanding of the molecular genetics of Huntington's disease (HD), nothing is known of the factor responsible for neuronal degeneration in HD.

Several animal models have been developed using neurotoxic agents; of these, intrastriatal injection of quinolinic acid (QA) provides the closest comparison to the cell losses found in HD. As well as being an "excitotoxin" of the glutamate receptor complex, QA is an endogenous metabolite of tryptophan and has been shown in animal studies to increase in concert with another metabolite, serotonin (5-hydroxytryptamine, 5HT) after tryptophan loading. We have found tryptophan levels to be unchanged in the HD striatum, but 5HT and 5-hydroxyindoleacetic acid concentrations are increased in many regions of the brain, including the striatum, which may be evidence for an abnormality of tryptophan metabolism.

Using a specific mass-spectrometric assay, we have determined $\mathrm{QA}$ in post mortem brain tissue from patients with HD and control subjects. In this preliminary study, concentrations of $\mathrm{QA}$ were found to be unchanged above normal in the HD putamen. These post mortem studies do not exclude the possibility of neurotoxic effects due to QA occurring in the living patient. Nevertheless the results suggest that an increase in brain concentrations is probably not responsible for neuronal degeneration in HD.

\section{HAND-GRIP DYNAMOMETRY IN PARKINSON'S} DISEASE

L Stacey, EJ Bassey, JH Hillier, RB Godwin-Austen, AT Birmingham. University of Nottingham Medical School and University Hospital, Nottingham

Hand-grip strength has been used by others as an index of post-operative recovery and in the assessment of drug efficacy in rheumatoid arthritis. We report the use of a hand-grip dynamometer in a comparison of the force-time curve of hand-grip in a group of patients with Parkinson's disease (PD) with a group of patients without the disease.

Nineteen out-patients with Parkinson's disease (15 males, age range 54-74 years; four females, age range 50-74) and 21 surgical out-patients ( 17 males, age range $50-76$ years; four females, age range 58-76) were measured during routine out-patient visits.

Maximal voluntary hand-grip force (MVC) and force-time data MVC were assessed for both hands using a standard procedure. The force-time curves were stored on floppy disk using a BBC microcomputer. From the 5 second MVC grip, the rate of rise, the fatigue rate during maintenance and the rate of release from the grip were measured. Display on a VDU of a target force of $20 \%$ MVC to be maintained for 15 seconds was also part of the test (based on M.I.E. software).

There were no differences between PD and normal patients (Mann-Whitney and Wilcoxon tests) for any of the measurements, except that the \% time on-target for a 15 second maintenance of a $20 \%$ MVC target force was $21 \%$ poorer for the PD patients than the normal patients $(p<0.02)$. Maintenance of the force-time curve was smooth for the normal patients but there was superimposition of a low frequency tremor for the PD patients.
A NEW ELECTRODIAGNOSTIC METHOD FOR THE DETECTION AND LOCALISATION OF NERVE ENTRAPMENT

WN Macleod. Royal Infirmary, Dundee

A method is described for detecting median nerve (MN) dysfunction in the carpal tunnel $\square$ (CT) by analysis of F response patterns. In each subject $M$ and $F$ responses were $\stackrel{\rho}{\supset}$ recorded from one site on abductor pollicis brevis (APB) stimulating (a) $\mathrm{MN}$ at the proximal edge of the CT, (b) $\mathrm{MN}$ at the distal edge of the CT, (c) ulnar nerve at the $\stackrel{\vec{P}}{\rightarrow}$ wrist. The palm stimulus was applied where $\overline{0}$ an $\mathbf{M} \geqslant \mathbf{M}$ evoked by the proximal $M N$ 들 supramaximal stimulus. One hundred $F \overline{\bar{\omega}}$ sweeps were recorded on an electro- $\vec{D}$ myograph from each stimulus site and two measurements made: (1) the \% Repeater Fwave $(\% R F)$ value $=$ total number of same- $\vec{O}$ latency, same-shape F's expressed as \% of total number of F's $\geqslant 40 \mu \mathrm{V},(2)$ the No F $\vec{\omega}$ response (NFR) value $=$ number of $F \stackrel{\circ}{\circ}$ sweeps where no $F \geqslant 40 \mu \mathrm{V}$ present.

Forty-one median and 41 ulnar nerves were examined in 24 asymptomatic volun- or teers, mean age 36 (19-68) and 11 median and ulnar nerves from 11 patients with $M \notin \overrightarrow{0}$ dysfunction detected by sensory and moto $\vec{\omega}$ nerve conduction studies and EMG, meat Gु age $39(31-48)$.

Per cent RF value at the wrist was $8 \frac{8}{\partial}$ (mean), (SD 8.1), and 8.3 (6.6) in the palre In controls NFR value at the wrist was $15 \hat{\beta}$ $(12.8)$, and $17.5(14.4)$ in the palm.

Mean difference in \%RF value across the CT was $-0 \cdot 2(7 \cdot 1)$, range +15 to -23 .

$\%$ RF in MN lesions either went from values in the control range to higher values $\infty$ from above to below the CT (e.g. $27 \%$ to $\square$ $100 \%$ ) or were abnormal at both sites, some- 웅 times increasing in abnormality distally ( $\%$ RF values were higher than upper value $\overline{0}$ of control range). Inadvertent activation of

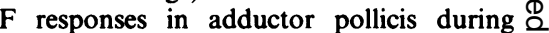
palmar stimulation was not found to be $\mathrm{a} \overrightarrow{\vec{F}}$ significant drawback.

\%RF values obtained by stimulating $\frac{3}{3}$ above and below a median nerve entrapment can detect and localise the site of the nerve lesion.

HEADACHE IN AN ACCIDENT AND EMERGENCY DEPARTMENT

DI Fodden, PL Milsom, RC Peatfield. 음 Pinderfields General Hospital, Wakefield

We have surveyed all patients complaining of headache attending the Accident and $S$ Emergency Department at St James's Hos- N 
pital, Leeds, over a 6 month period, examining the hospital in-patient notes and securing GP follow-up notes for the remainder. Of the 130 patients, 24 proved untraceable despite diligent efforts leaving full follow-up data on 106. Thirty-eight $(36 \%)$ were male and $40(38 \%)$ were GP referrals. Twenty-eight of the patients proved to have non-specific headaches, 20 had migraine (including cluster headaches), 17 were related to general medical problems, 14 were considered to be psychogenic and 10 were associated with generalised febrile illnesses. There were four patients with cerebral haemorrhages and five with subarachnoid haemorrhages, two known to have tumours (each attending twice), one with an arteriovenous malformation and one with benign intracranial hypertension, and two cases of meningitis. All but three of the last 15 patients had abnormal neurological signs, and in these three serious pathology was evident from the history alone.

We conclude that structural causes of headaches are more common than reported in other published series from GP, Neurological, and Headache Clinics, and the presence of abnormal neurological signs demands admission. No patient in whom a "benign" diagnosis was made initially ultimately had any pathology justifying active treatment.

FRIEDREICH'S ATAXIA IN IRELAND; A CLINICAL AND INVESTIGATIVE STUDY EA Martin, M McLaughlin, R Clarke, I Graham, D McInerney, G Dean. Adelaide Hospital, Dublin

Five patients with a diagnosis of Friedreich's ataxia (FA) were examined and investigated by neurological, cardiac, psychological examinations and the following: Nerve conduction velocities; Holter monitoring; ECG; ECHO; radiology of skull, chest, spine, pelvis, hands, feet; General Health Questionnaire (GHQ); Clinical Interview Schedule (CIS). Nine patients did not meet diagnostic criteria and were excluded.

The average age (total 36 patients) was
23.6 years (range 6-67 years). Mean age of onset was 10.4 years (range 3-25 years). Average duration of FA was 13.3 years (range 1-47 years). Average duration of disease before becoming chairbound was 13.6 years. Sixty-six per cent had abnormal ECGs and $64 \%$ abnormal ECHOs. Scoliosis was almost universally present and more severe in females than males. Eleven per cent had mild to moderate deafness and $6 \%$ were diabetic. The mean median MCV was $54.3 \mathrm{~m} / \mathrm{s}$ (range $49-65 \mathrm{~m} / \mathrm{s}$ ). Thirty-three per cent scored significantly on the GHQ.

Absence of lower limb reflexes is usually reckoned essential for a diagnosis of Friedreich's ataxia. Seven patients in this study retained some degree of lower limb deep reflexes. They met all other diagnostic criteria, and otherwise were indistinguishable on clinical or investigative grounds. All had a close relative with classical FA.

This study indicates a need for modification of current diagnostic criteria in relation to lower limb areflexia in Friedreich's ataxia.

MUSCLE FUNCTION TESTING IN POLYMYOSITIS CM Wiles, Y Karni, J Nicklin. National Hospitals for Nervous Diseases, London

Since 1982,512 patients with peripheral neuromuscular disease have been assessed in a muscle function laboratory using strength measurements, a standard performance scale, timed walking over $\mathbf{3 0}$ metres and an analogue scale of wellbeing. Of 42 patients with polymyositis 17 (six male, 11 female, age range 22 to 68 years) have been monitored for more than a year (mean $2 \cdot 94$, range 1.1-4.7 years). All received immunosuppressive medication.

Nine patients showed significant improvement in strength (seven into the normal range) of whom two died. Five patients showed no improvement in strength, of whom one died, and three became weaker. Alterations in strength were associated with changes in walking time and functional performance except where another factor like weight change or systemic illness intervened. However, large changes in functional ability only occurred when muscle strength had fallen substan- $Z$ tially below the normal range. Plasma cre- $\stackrel{\mathbb{D}}{\subseteq}$ atine kinase (CK) levels correlated poorly $\bar{O}$ with muscle strength. Improvements in strength in individuals were usually associ- $\bar{C}$ ated with a decline in CK and relapses of the $\square$ disease with a rise.

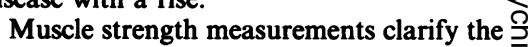
response to treatment in polymyositis and are essential (with performance tests) in maintaining a perspective of progress over long periods of follow up.

RESPIRATORY ARREST: A COMPLICATION OF CEREBELLAR ECTOPIA IN ADULTS DR Fish, RS Howard, CM Wiles, L Symon. $\stackrel{ه}{\varnothing}$ St Thomas' Hospital, London

Two women aged 54 and 63 years with cere- $\overrightarrow{0}$ bellar ectopia presented with respiratory $\overrightarrow{\vec{\omega}}$ arrest. Both patients had shown neurological abnormalities for some years before presentation and these included horizontal and downbeat nystagmus, cerebellar ir ataxia and paraparesis. In both patients. there were premonitary symptoms of hypo- $\overrightarrow{0}$ ventilation or sleep apnoea and the radio logical and operative findings suggestef cerebellar ectopia with anterior compression of the brainstem and venterolateral upp cervical cord. Following surgical decort pression of the foramen magnum both $\bigcirc$ patients became self ventilating and nog lead independent lives in the community.

A primary abnormality of ventilato control may have led to apnoea in both $\overrightarrow{0}$ patients due to involvement of the brainstem $\infty$ and/or anterior pathways subserving the automatic (metabolic) control of breathing.

Respiratory complications of cerebellar ectopia may be occult, therefore respiratory $\bar{\partial}$ function testing and blood gas analysis should be routinely considered in these $\overparen{D}$ patients and sleep studies may be of value.

The risk of sudden respiratory arrest, as well as gradual respiratory deterioration, 3 must be considered in planning the management of patients in whom a diagnosis of cerebellar ectopia has been made and this potentially treatable condition should be considered in patients with unexplained? respiratory arrest. 